\title{
3D quantitative analysis of diffusion-weighted imaging for predicting the malignant potential of intraductal papillary mucinous neoplasms of the pancreas
}

\author{
Takao Igarashi ${ }^{A, B, C, D, E, F}$, Megumi Shiraishi ${ }^{B}$, Ken Watanabe ${ }^{B}$, Kazuyoshi Ohki ${ }^{D}$, Shinsuke Takenaga ${ }^{E}$, \\ Hirokazu Ashida ${ }^{\mathrm{E}}$, Hiroya Ojiri ${ }^{\mathrm{E}}$ \\ Department of Radiology, The Jikei University School of Medicine, Minato-ku, Tokyo, Japan
}

\section{Abstract}

Purpose: To investigate the predictors of intraductal papillary mucinous neoplasms of the pancreas (IPMNs) with high-grade dysplasia, using 2-dimensional (2D) analysis and 3-dimensional (3D) volume-of-interest-based apparent diffusion coefficient (ADC) histogram analysis.

Material and methods: The data of 45 patients with histopathologically confirmed IPMNs with high-grade or lowgrade dysplasia were retrospectively assessed. The $2 \mathrm{D}$ analysis included lesion-to-spinal cord signal intensity ratio (LSR), minimum $\mathrm{ADC}$ value $\left(\mathrm{ADC}_{\text {min }}\right)$, and mean $\mathrm{ADC}$ value $\left(\mathrm{ADC}_{\text {mean }}\right)$. The $3 \mathrm{D}$ analysis included the overall mean $\left(\mathrm{ADC}_{\text {overall mean }}\right)$, mean of the bottom $10^{\text {th }}$ percentile $\left(\mathrm{ADC}_{\text {mean }-10}\right)$, mean of the bottom $10-25^{\text {th }}$ percentile $\left(\mathrm{ADC}_{\text {mean } 10-25}\right)$, mean of the bottom $25-50^{\text {th }}$ percentile $\left(\mathrm{ADC}_{\text {mean25-50 }}\right)$, skewness $\left(\mathrm{ADC}_{\text {skewness }}\right)$, kurtosis $\left(\mathrm{ADC}_{\text {kurtosis }}\right)$, and entropy $\left(\mathrm{ADC}_{\text {entropy }}\right)$. Diagnostic performance was compared by analysing the area under the receiver operating characteristic curve (AUC). Inter-rater reliability was assessed by blinded evaluation using the intraclass correlation coefficient.

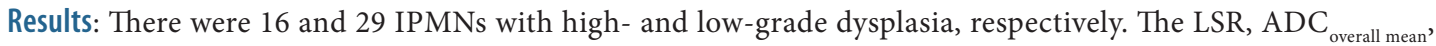
$\mathrm{ADC}_{\text {mean0-10 }}, \mathrm{ADC}_{\text {mean 10-25, }}, \mathrm{ADC}_{\text {mean25-50, }}$, and $\mathrm{ADC}_{\text {entropy }}$ showed significant between-group differences $(\mathrm{AUC}=72-93 \%$; $p<0.05)$. Inter-rater reliability assessment showed almost perfect agreement for LSR and substantial agreement for $\mathrm{ADC}_{\text {overall mean }}$ and $\mathrm{ADC}_{\text {entropy }}$ Multivariate logistic regression showed that ADCoverall mean and ADCentropy were significant independent predictors of malignancy $(p<0.05)$, with diagnostic accuracies of $80 \%$ and $73 \%$, respectively. Conclusion: $\mathrm{ADC}_{\text {overall mean }}$ and $\mathrm{ADC}_{\text {entropy }}$ from 3D analysis may assist in predicting IPMNs with high-grade dysplasia. Key words: diffusion magnetic resonance imaging, mucinous carcinoma, pancreatic duct, pancreatic intraductal neoplasms.

\section{Introduction}

Intraductal papillary mucinous neoplasms of the pancreas (IPMNs) were first recognized as an entity in the $1980 \mathrm{~s}$ $[1,2]$ and are increasingly detected due to widespread use of imaging technology. IPMNs are classified into those with low-grade dysplasia, with high-grade dysplasia, and with invasive carcinoma [3]. High-grade IPMNs are histopathologically considered as an early cancer stage. The 5-year survival rates for non-invasive and invasive IPMNs are $88-100 \%[4,5]$ and $34-42 \%[6,7]$, respectively. Thus, high-grade dysplastic IPMNs may be a potentially curable pancreatic cancer if treated before becoming invasive. The favourable prognostic factors for IPMN-associated carcinoma (compared with common-type pancreatic ductal carcinoma) are lower rates of advanced T stage dis-

Correspondence address:

Dr. Takao Igarashi, Department of Radiology, The Jikei University School of Medicine 3-25-8, Nishi-Shimbashi, Minato-ku, Tokyo 105-8461, Japan, e-mail: igarashi-t@jikei.ac.jp

Authors' contribution:

A Study design · B Data collection - C Statistical analysis · D Data interpretation - E Manuscript preparation · F Literature search · G Funds collection 
ease, lymph node metastasis, high tumour grade, positive resection margin, and perineural and vascular invasion [8]. These factors, associated with imaging findings, can be assessed using preoperative magnetic resonance imaging (MRI); therefore, MRI findings could be significant for the evaluation of the malignant potential of IPMN, particularly prior to progression to invasive carcinoma. Differentiating potentially malignant from benign neoplasms before this stage can help guide clinical strategy, in particular if a non-invasive screening technique can be used.

MRI with magnetic resonance cholangiopancreatography (MRCP) is a useful imaging modality for evaluating the malignant potential and surgical resectability of IPMNs [9]. More specifically, diffusion-weighted imaging (DWI) is considered effective for the diagnosis of malignant IPMNs [10-12], but with limited evidence. Malignant pancreatic tumours generally show higher signal intensities on DWI and lower apparent diffusion coefficient (ADC) values than do benign tumours $[13,14]$. DWI performance in evaluating the malignant potential of cystic pancreatic lesions is comparable to that of contrast-enhanced magnetic resonance imaging (MRI) $[12,15]$. Furthermore, the combined use of DWI and MRCP, and the use of the minimum $\left(\mathrm{ADC}_{\text {min }}\right)$ and mean $\mathrm{ADC}\left(\mathrm{ADC}_{\text {mean }}\right)$ values, are effective for diagnosing malignant IPMNs and predicting the risk for IPMNs with invasive carcinoma $[10,12,13]$. The lesionto-spinal cord signal intensity ratio (LSR) was recently proposed as a useful measure for predicting malignancy. LSR is a convenient semiquantitative variable that can be calculated on DWI [16-19]. LSR is comparable to the ADC measurement method and can be used with any MRI scanner $[17,18]$. In general, the conventional method, using 2-dimensional region-of-interest (ROI)-based analysis (hereafter referred to as 2D analysis), may be highly representative, is less amenable to manual ROI variability, and is less time-consuming. Conversely, whole-lesion ADChistogram analysis using 3-dimensional volume-of-interest (VOI) (hereafter referred to as 3D analysis) of the entire IPMN is also useful for assessing the malignant potential of pancreatic IPMNs [20]. Pixel-by-pixel analysis of a slice in a tumour can reveal tumour heterogeneity [20].

However, the best method for predicting malignant potential in early-stage IPMNs remains unclear. Moreover, differentiating IPMNs with high-grade dysplasia from those with low-grade dysplasia using non-invasive techniques may help optimize clinical strategies. Thus, this study investigated the predictors of IPMNs with highgrade dysplasia using $2 \mathrm{D}$ and $3 \mathrm{D}$ analyses based on DWI.

\section{Material and methods}

\section{Patients}

This retrospective study was approved by the relevant review committee, and the procedures followed were in accordance with the Helsinki Declaration of 1975, as revised in 2000. The requirement for written informed consent was waived by the relevant institutional review board because of the retrospective study design. The study included patients who were diagnosed with IPMN of the pancreas, with a maximum mass diameter $\geq 1 \mathrm{~cm}$, based on post-surgical histopathological reports, between 1 June 2009 and 30 September 2019. All data were retrospectively identified from our institutional imaging database. We excluded patients who did not undergo MRI within 6 months pre-surgery, in whom IPMN with a maximum diameter $<1 \mathrm{~cm}$ was an incidental finding (not indicated for surgery), and who were histopathologically diagnosed with IPMNs with invasive carcinoma. No patient underwent fine needle aspiration (FNA) or chemotherapy before MRI and surgery.

\section{Magnetic resonance imaging technique}

All patients underwent MRI on a 1.5-Tesla MR unit (Magnetom Avanto or Symphony, Siemens Medical Solutions, Erlangen, Germany [ $n=39]$ ) with a 6-channel body matrix coil, or a 3.0-Tesla MR unit (Skyra, Siemens Medical Solutions, Erlangen, Germany $[n=6])$ with an 18 -channel body coil.

T1-weighted breath-hold spoiled gradient-recalledecho (GRE) in-phase and opposed-phase images, 3D-fatsuppressed T1-weighted GRE sequences with Cartesian sampling (volumetric interpolated breath-hold examination on Siemens Healthcare MRI systems), T2-weighted fast-spin-echo images, DW images with low and high $b$-values ( $b=50$ and $800 \mathrm{~s} / \mathrm{mm}^{2}$, respectively), and halfFourier acquisition single-shot turbo spin-echo sequences were obtained. ADC maps were automatically generated using the operating console. Respiratory-triggered 3D-T2-weighted MRCP with maximum intensity projection was performed. Details of imaging parameters in this study are summarized in Supplementary Table 1.

\section{Histopathological analysis}

Determination of the IPMN grade (low-grade dysplasia, high-grade dysplasia, and invasive carcinoma) was based on the highest-grade focus. The presence or absence of invasive carcinoma was determined based on histopathological examinations in all cases. Lesions with minimal invasion, i.e. a few lesions with only a few tumour glands communicating with the intraductal carcinoma components, were also considered invasive carcinoma.

\section{Image analysis}

A radiologist (17 years of MRI experience) retrospectively reviewed all MR images. The reader was not blinded to preoperative reports of computed tomography, MRI, endoscopic ultrasonography, or clinical and histopatho- 
Table 1. Clinical characteristics of patients in our cohort

\begin{tabular}{|c|c|c|c|}
\hline Factor & $\begin{array}{l}\text { IPMNs with high-grade dysplasia } \\
\qquad n=16\end{array}$ & $\begin{array}{l}\text { IPMNs with low-grade dysplasia } \\
\qquad n=29\end{array}$ & $p$-value \\
\hline Age (years) ${ }^{\mathrm{a}}$ & $74.4 \pm 5.62$ & $65.8 \pm 9.92$ & 0.002 \\
\hline Sex: male (\%) & $14(88)$ & $19(66)$ & 0.16 \\
\hline \multicolumn{4}{|l|}{ Location } \\
\hline Head & 10 & 15 & \multirow[t]{2}{*}{0.54} \\
\hline Body, tail & 6 & 14 & \\
\hline \multicolumn{4}{|l|}{ IPMN lesion type } \\
\hline Branch & 6 & 22 & \multirow[t]{3}{*}{0.02} \\
\hline Mixed & 6 & 6 & \\
\hline MPD & 4 & 1 & \\
\hline \multicolumn{4}{|l|}{ Surgical procedure } \\
\hline Pancreaticoduodenectomy & 10 & 17 & \multirow[t]{4}{*}{0.99} \\
\hline Distal pancreatectomy & 6 & 10 & \\
\hline Total pancreatectomy & 0 & 1 & \\
\hline Partial pancreatectomy & 0 & 1 & \\
\hline Pathological size $(\mathrm{mm})^{\mathrm{a}, \mathrm{b}}$ & $50.4 \pm 27.5$ & $35.6 \pm 21.5$ & 0.03 \\
\hline
\end{tabular}

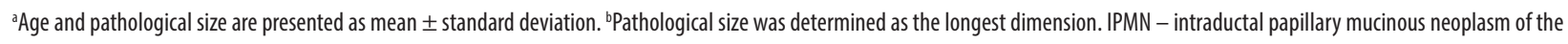
pancreas, MPD - main pancreatic duct

logical examinations. With reference to the revised International Consensus Guidelines for the Management of IPMN of the Pancreas, 2017 [21], preoperative MRI features were evaluated on a picture archiving and communication system (PACS) monitor.

Binary MRI variables were evaluated as present or absent, as follows: enhancing mural nodule $>5 \mathrm{~mm}$, main pancreatic duct (MPD) size $\geq 10 \mathrm{~mm}$, cyst size $\geq 3 \mathrm{~cm}$, enhancing mural nodule $<5 \mathrm{~mm}$, thickened enhancing cyst wall $\geq 3 \mathrm{~mm}$, MPD size 5-9 mm, abrupt MPD calibre change, and lymphadenopathy. An electronic calliper, included in the PACS program, was used to obtain measurements from any axial or coronal image. If several cystic lesions were present, the lesion with the largest wall and septal thickness and mural nodule was used for analysis. A mural nodule was defined as any solid nodule in the MPD or branch duct of a well-circumscribed tissue lesion surrounded by a duct wall. Lymphadenopathy was considered "present" when the short axis diameter exceeded $10 \mathrm{~mm}$.

\section{Blinded evaluation during 2D and $3 \mathrm{D}$ analyses}

All data except information regarding the target lesion were anonymized. One blinded radiologist (Reader 1: 4 years' experience) analysed body MR images to determine LSR, $\mathrm{ADC}_{\text {min }}$, and $\mathrm{ADC}_{\text {mean }}$ of all lesions for $2 \mathrm{D}$ analysis. Figure 1 illustrates the 2D analysis methods used. LSR was measured on a DW image with a diffusion gradient of $b=800 \mathrm{~s} / \mathrm{mm}^{2}$ and calculated as follows: mean signal intensity of the lesion on DWI/maximum signal intensity of the spinal cord on DWI. ADC values were measured after establishing LSR. ROI in the spinal cord on DWI was located on a slice of the lumbar enlargement because the tissue volume was larger in this area than in other spinal regions, thus facilitating ROI localization. ROIs of the same size were set at the same location as that of the pancreatic lesion on each DW image and ADC map. ROIs in the pancreatic lesion on both the DW image and ADC map were round or oval and covered the solid component or septum/cyst wall as much as possible, excluding a partial-volume phenomenon. The highest mean signal intensity of the lesion on DWI, the lowest $\mathrm{ADC}_{\text {min }}$, and the lowest $\mathrm{ADC}_{\text {mean }}$ among the 3 measurements were adopted for analyses.

The same lesions evaluated by $2 \mathrm{D}$ analysis were subjected to $3 \mathrm{D}$ analysis by the same radiologist. For each patient, a 3D-VOI was placed on the area recognized as a mass with solid components on the ADC map using specialized application software (FireVoxel; https:// wp.nyu.edu/firevoxel). ADC values were constructed into a histogram, which was used to obtain parameters of the first-order statistical properties of the image. The overall mean $\left(\mathrm{ADC}_{\text {overall mean }}\right)$, mean of the bottom $10^{\text {th }}$ percentile $\left(\mathrm{ADC}_{\text {mean } 0-10}\right)$, mean of the bottom $10-25^{\text {th }}$ percentile $\left(\mathrm{ADC}_{\text {mean 10-25 }}\right)$, mean of the bottom $25-50^{\text {th }}$ percentile $\left(\mathrm{ADC}_{\text {mean25-50 }}\right)$, skewness $\left(\mathrm{ADC}_{\text {skewness }}\right)$, kurtosis $\left(\mathrm{ADC}_{\text {kurtosis }}\right)$, and entropy $\left(\mathrm{ADC}_{\text {entropy }}\right)$ [22] were determined. Figure 2 illustrates the 3D analysis methods used. To investigate the reproducibility of the results of Reader 1 , another blinded radiologist (Reader 2: 12 years' experience) performed $2 \mathrm{D}$ and $3 \mathrm{D}$ analyses using the same 

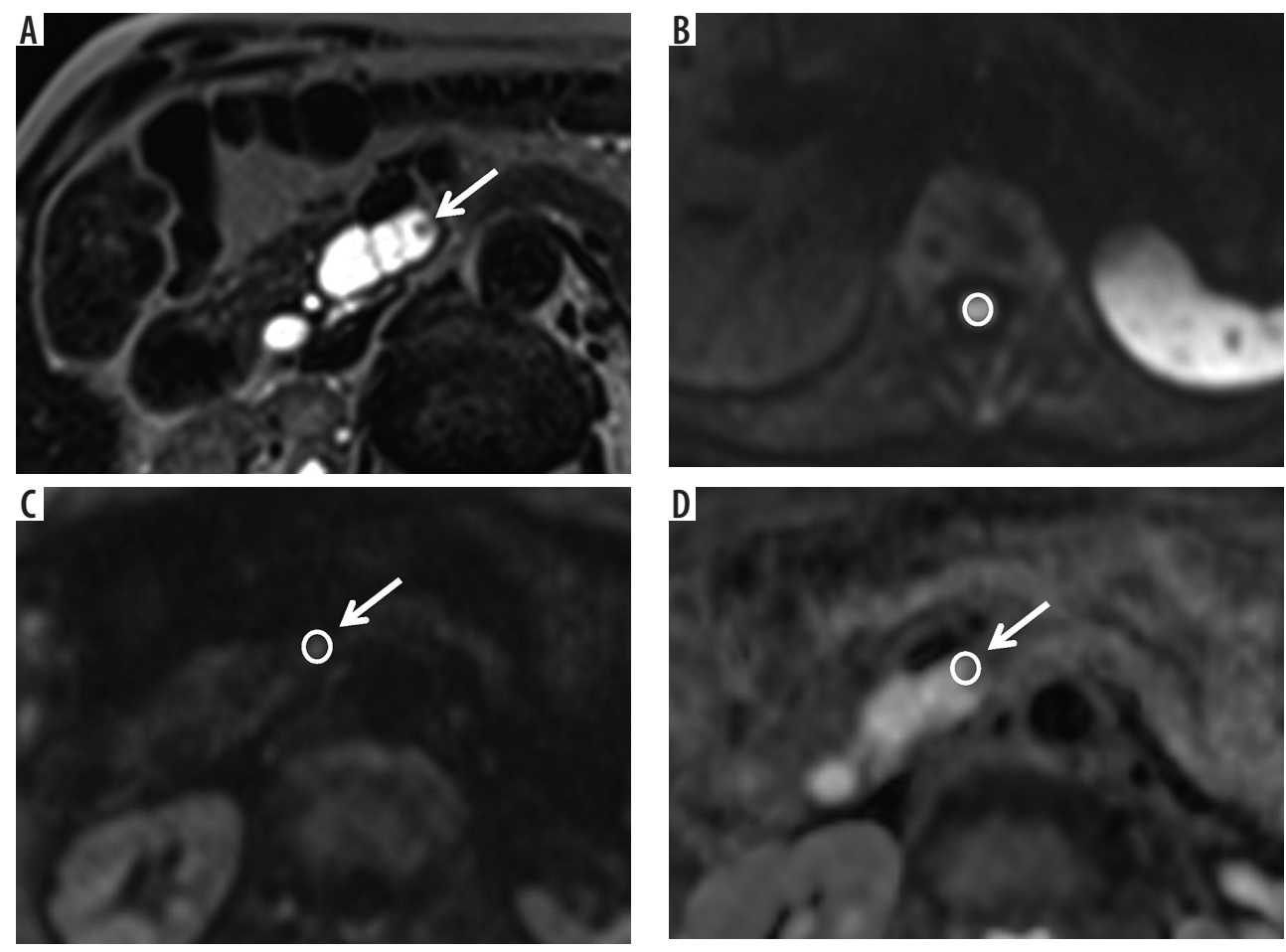

Figure 1. Example of 2D analysis method. Sample of 1.5-Tesla magnetic resonance images of a 76-year-old man with branch-type intraductal papillary mucinous neoplasm of the pancreas (IPMN) with high-grade dysplasia in the head of the pancreas. A) Half-Fourier acquisition single-shot turbo spin-echo (HASTE) image. B-C) Diffusion-weighted (DW) image. D) Apparent diffusion coefficient (ADC) map. Mural nodule was identified in the branch-type IPMN on HASTE images (A). First, ROl in the spinal cord on DWI was located on a slice of the lumbar enlargement (B). Then, ROls of the same size were set at the same location as that of the pancreatic lesion on each DW image and $\operatorname{ADC}$ map $(C, D)$. Finally, the maximum signal intensity of the spinal cord on DWI, the highest mean signal intensity of the lesion on $D W I$, the lowest $A D C_{\text {min }^{\prime}}$ and the lowest $A D C_{\text {mean }}$ among the 3 measurements were adopted for analyses. Area of ROI, LSR, $A D C_{\text {min' }}$ and $A D C_{\text {mean }}$ were $52\left(\mathrm{~mm}^{2}\right), 0.29,1.354\left(\times 10^{-3} \mathrm{~mm}^{2} / \mathrm{s}\right)$, and $2.201\left(\times 10^{-3} \mathrm{~mm}^{2} / \mathrm{s}\right)$, respectively

method. Reader 2 was blinded to the results of Reader 1 and the clinical and histopathological information.

\section{Statistical analysis}

The Mann-Whitney $U$ and Fisher's exact tests were used to compare continuous and binary variables, respectively. All continuous variables measured by Reader 1 were used for statistical assessment of 2D and 3D analyses. All continuous variables measured by Reader 2 were used for inter-rater reliability assessment. The diagnostic performances of continuous variables were estimated using receiver operating characteristic (ROC) curves and evaluated by comparing the area under the ROC curve (AUC), considering histopathological examination as the reference standard. Inter-rater reliability was assessed using intraclass correlation coefficients (ICCs). ICCs of 0.81-1.0, 0.61-0.80, 0.41-0.60, 0.21-0.40, and $\leq 0.20$ represented almost perfect, substantial, moderate, fair, and slight agreement, respectively. Multivariate logistic regression analysis was performed with continuous variables of $2 \mathrm{D}$ and $3 \mathrm{D}$ analyses to determine the independent predictors of IPMNs with high-grade dysplasia. Continuous variables that showed significant association with IPMNs with high-grade dysplasia in univariate analysis and had almost perfect or substantial agreement in the inter-rater reliability assessment were included in the multivariate logistic regression analysis. Diagnostic accuracy was calculated based on cut-off values chosen to maximize the Youden index on the ROC analysis and assessed for the continuous variables in the multivariate analysis. A $p$-value of $<0.05$ was considered statistically significant. All statistical analyses were performed using R software (The R Project for Statistical Computing, www.r-project.org, version 3.3.0) and SPSS (ver. 25; IBM Corp., Armonk, NY).

\section{Results}

The participant selection process is depicted in Figure 3. This study included 45 patients with histopathologically confirmed IPMNs with high- $(n=16)$ or low-grade dysplasia $(n=29)$ (Figures 4 and 5). Compared with patients with IPMNs with low-grade dysplasia, those with highgrade dysplasia were older and had larger tumours as determined by the largest tumour diameter $(p<0.05$; Table 1). Among MRI features, only an abrupt calibre change in MPD was significantly associated with IPMNs with high-grade dysplasia in univariate analysis $(p<0.05$; Table 2). The results of $2 \mathrm{D}$ and $3 \mathrm{D}$ analyses are shown in Table 3. Box and whisker plots of 2D and 3D analyses are shown in Figure 6. The ROIs of the 2D analysis 

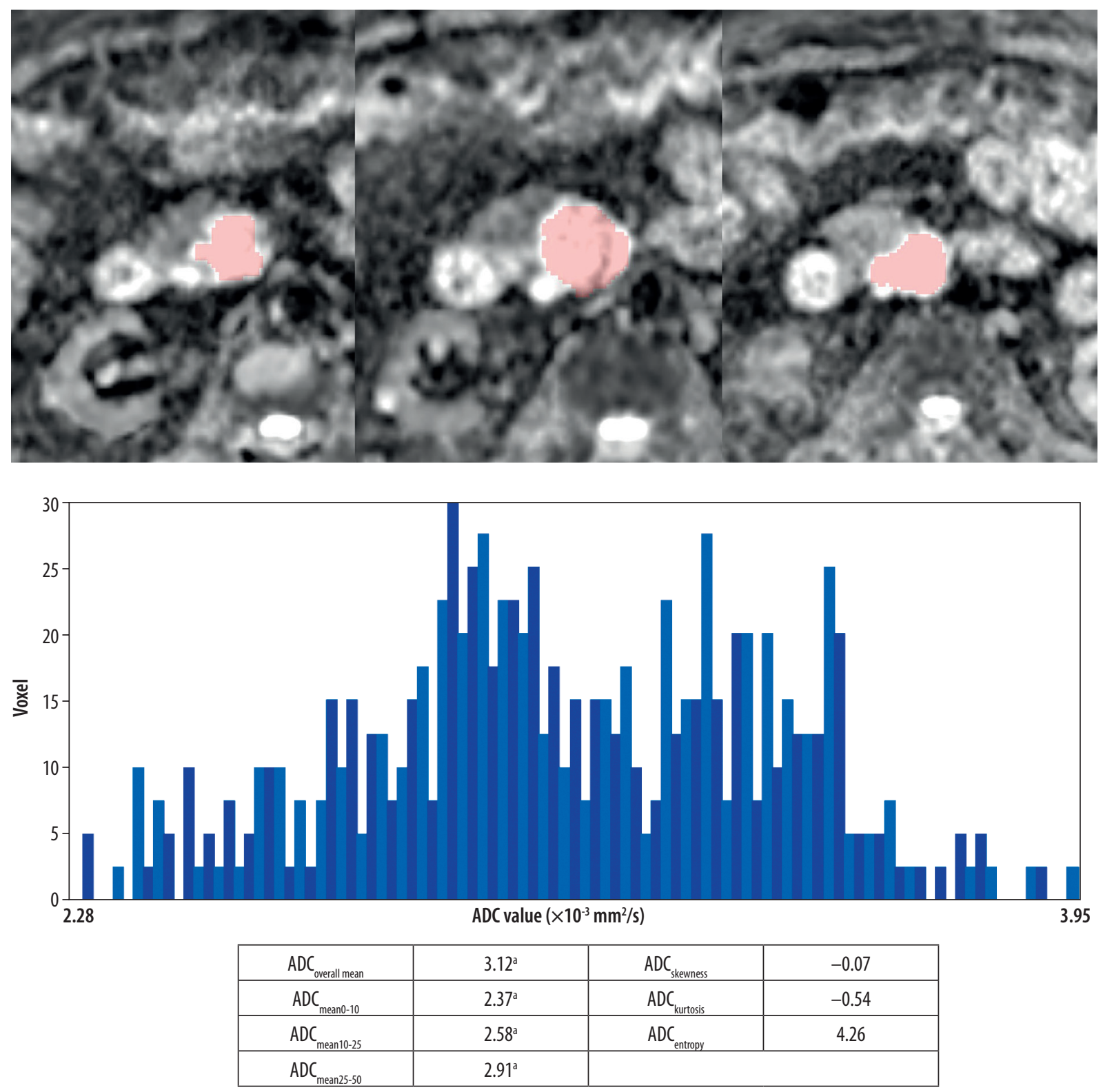

Figure 2. Example of 3D analysis method. Sample of 1.5-Tesla magnetic resonance images of a 79-year-old man with branch-type intraductal papillary mucinous neoplasm of the pancreas (IPMN) with high-grade dysplasia in the head of the pancreas. A 3D-VOI was placed on the area diagnosed as a mass with solid components on the apparent diffusion coefficient (ADC) map (areas marked in pink). All slices in which visible areas with measurable $A D C$ values within the lesion occurred, were included. The $A D C$ values were then constructed into a histogram as follows: Median $A D C$ value $=3.08\left(\times 10^{-3} \mathrm{~mm}^{2} / \mathrm{s}\right)$, voxel count $=390$, volume $=8.55 \mathrm{~cm}^{3}$.

$(p=0.97)$ and the VOIs of the $3 \mathrm{D}$ analysis $(p=0.46)$ were not significantly different. The LSR (mean value \pm standard deviation) was $0.3 \pm 0.1$ for IPMNs with highgrade dysplasia and $0.23 \pm 0.09$ for IPMNs with lowgrade dysplasia (AUC: 72\%; $p<0.05$ ). $\mathrm{ADC}_{\text {overall mean }}$, $\mathrm{AD}$ $\mathrm{C}_{\text {mean0-10 }}, \mathrm{ADC}_{\text {mean10-25 }}, \mathrm{ADC}_{\text {mean25-50, }}$, and $\mathrm{ADC}_{\text {entropy }}$ showed significant between-group differences $(A U C=72-93 \%$; $p<0.05)$. Inter-rater reliability analysis showed almost perfect agreement for LSR and VOIs, and substantial agreement for $\mathrm{ADC}_{\text {overall mean }}$ and $\mathrm{ADC}_{\text {entropy }}$ (Table 4). Multivariate logistic regression analysis showed that $\mathrm{ADC}_{\text {overall mean }}$ and $\mathrm{ADC}_{\text {entropy }}$ were significant independent predictors of IPMNs with high-grade dysplasia (Table 5).
The diagnostic accuracies of $\mathrm{ADC}_{\text {overall mean }}$ and $\mathrm{ADC}_{\text {entropy }}$ were $80 \%$ and $73 \%$, respectively (Table 5 ).

\section{Discussion}

The results of this study showed that $\mathrm{ADC}_{\text {overall mean }}$ and $\mathrm{ADC}_{\text {entropy }}$ were significant independent predictors of IPMNs with high-grade dysplasia. Because none of the patients with IPMNs underwent FNA prior to MRI and surgery, all surgeries were performed considering the possibility of malignant potential. Patients with lowgrade dysplasia presented more enhancing mural nodules than did patients monitored for suspected IPMNs 
Patients who were diagnosed as IPMN of the pancreas with a maximum diameter of $\geq 1 \mathrm{~cm}$ upon histopathological examination after surgery between 01/Jun/2009 and 30/Sep/2019

Patients who underwent MR imaging before surgery

Patients who underwent dynamic CT and/or EUS within 6 months before surgery, $n=80$

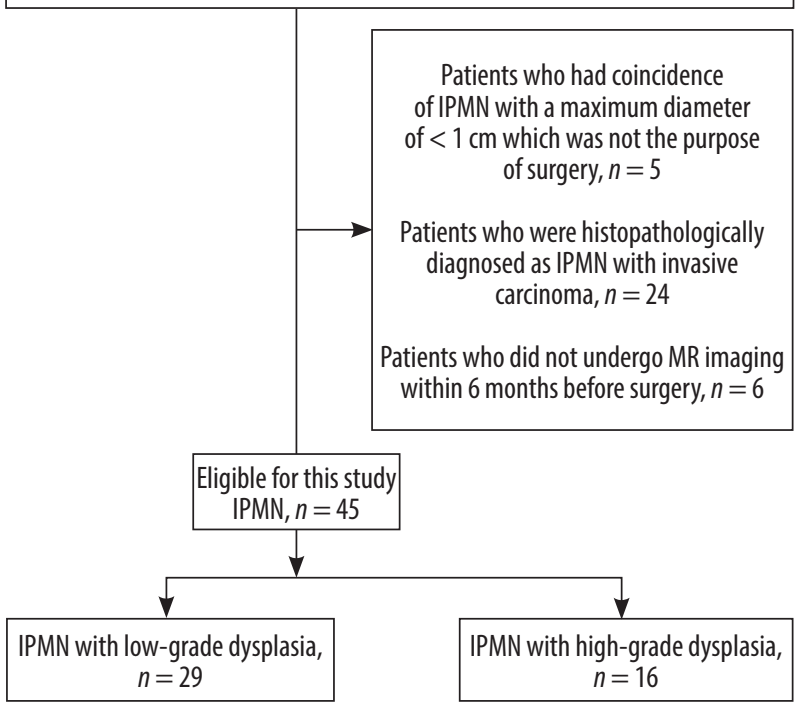

Figure 3. Flow diagram of the patient selection process. IPMN - intraductal papillary mucinous neoplasm of the pancreas, MR - magnetic resonance, CT - computed tomography, EUS - endoscopic ultrasonography with low-grade dysplasia in clinical practice. Consequently, enhancing mural nodules showed no significant association with IPMNs with high- and low-grade dysplasia in our univariate analysis. However, only abrupt calibre change in MPD was significantly associated with IPMNs with high-grade dysplasia. Our results indicated that differentiating IPMNs with high-grade dysplasia from those with low-grade dysplasia by analysing MRI features, based on the 2017 revised guidelines, might be difficult [21]

Traditionally, 2D analysis is performed using a value from within a manually set ROI on a single-slice image $[10,13]$. Theoretically, the most viable malignancy component can be located anywhere within the lesion; thus, $2 \mathrm{D}$ analysis is prone to subjective evaluation of lesion heterogeneity. Therefore, the inter-rater reliability for $2 \mathrm{D}$ analysis in this study showed fair agreement in all areas except for LSR. ADC value measurements in this study depended on the site chosen for setting the ROIs, which is highly reader-dependent and can cause reproducibility issues. Moreover, intralesional haemorrhage and fibrosis may manifest as blacked-out areas on T2-weighted images, which decreases the ADC value, irrespective of the presence of true restricted diffusion [17]. Conversely, LSR showed significant between-group differences and almost
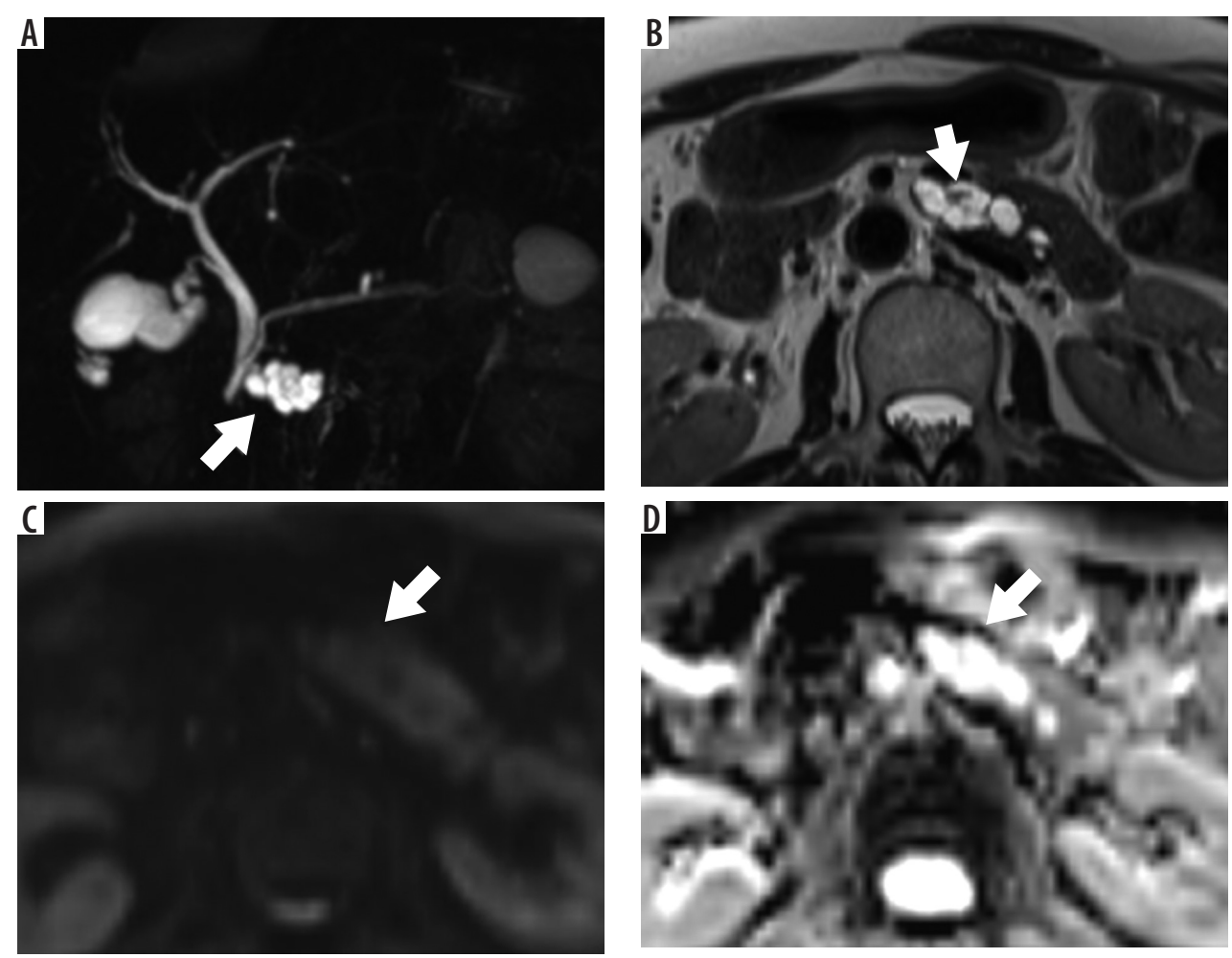

Figure 4. Sample of 3.0-Tesla magnetic resonance images in a 63-year-old woman with branch-type intraductal papillary mucinous neoplasm of the pancreas (IPMN) with low-grade dysplasia in the body of the pancreas. A - Maximum intensity projection image showing cystic lesion with lobulated shape and multi-septal structures (arrow). B - T2-weighted turbo spin echo image showing cystic lesion with a mural nodule ( $\geq 5 \mathrm{~mm}$; arrowhead). C - Diffusion-weighted image showing no obvious high signal intensity (arrow). D - No definite restricted region was detected on the apparent diffusion coefficient $(A D C)$ map (arrow). $A D C_{\text {overall mean }}$ and $A D C_{\text {entropy }}$ were 3.16 and $3.05\left(\times 10^{-3} \mathrm{~mm}^{2} / \mathrm{s}\right)$, respectively 

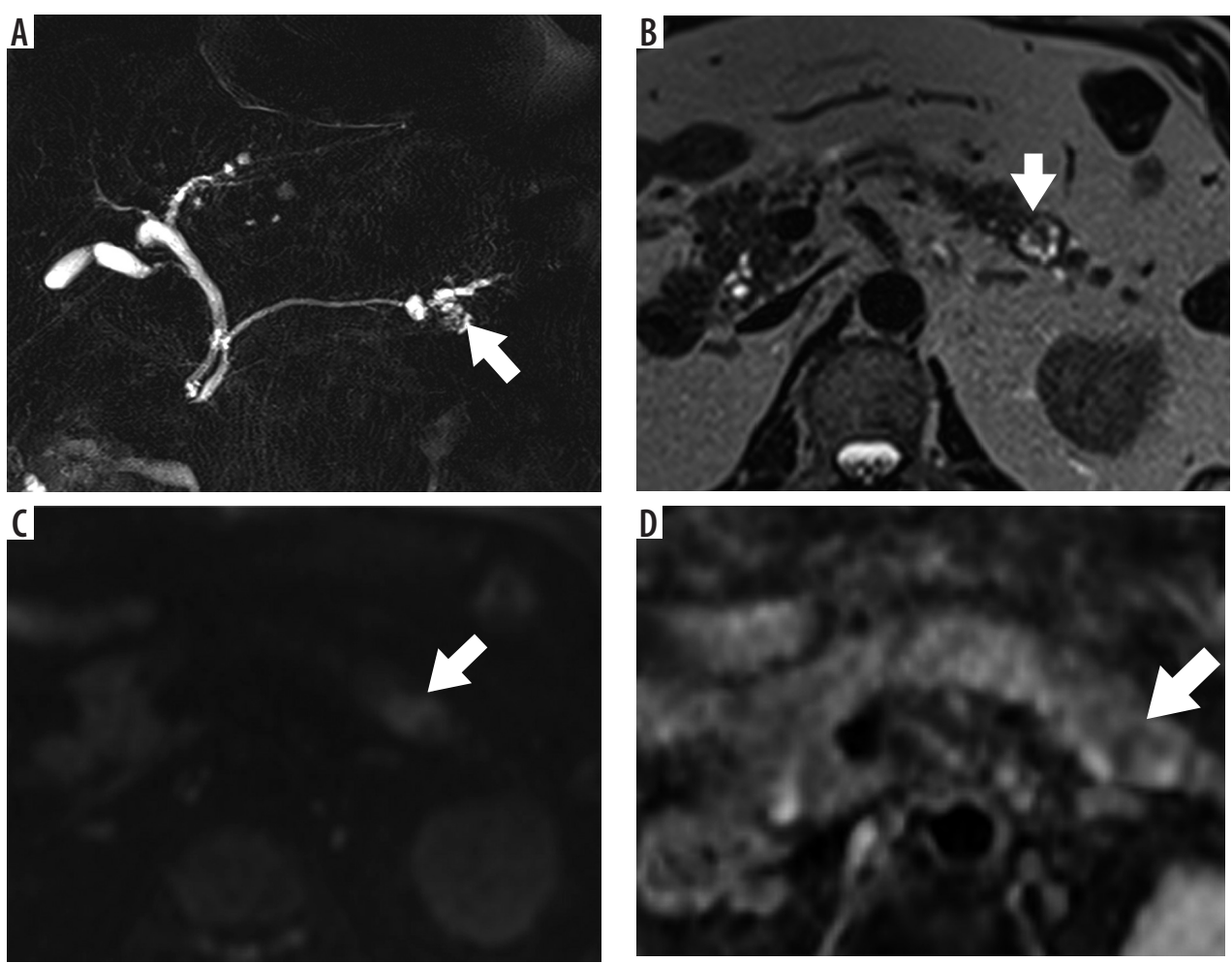

Figure 5. Sample of 1.5-Tesla magnetic resonance images in a 76-year-old man with non-invasive intraductal papillary mucinous neoplasm of the pancreas (IPMN) with high-grade dysplasia in the tail of the pancreas. A - Maximum intensity projection image showing cystic lesion with multi-septal structures (arrow). B - T2-weighted turbo spin echo image showing cystic lesion with a mural nodule (arrowhead). C - Diffusion-weighted image showing a high signal lesion (arrow). D - A partially low signal region was detected on the apparent diffusion coefficient (ADC) map, suggestive of restricted diffusion (arrow). $A D C_{\text {overall mean }}$ and $A D C_{\text {entropy }}$ were $1.88\left(\times 10^{-3} \mathrm{~mm}^{2} / \mathrm{s}\right)$ and 4.21 , respectively

Table 2. Magnetic resonance imaging features of patients with intraductal papillary mucinous neoplasm of the pancreas (IPMNs) with high- and with lowgrade dysplasia with reference to the revised International Consensus Guidelines for the Management of IPMN of the Pancreas, 2017

\begin{tabular}{|c|c|c|c|}
\hline Factor & $\begin{array}{l}\text { IPMNs with high-grade dysplasia } \\
\qquad n=16^{\mathrm{a}}\end{array}$ & $\begin{array}{l}\text { IPMNs with low-grade dysplasia } \\
\qquad n=29^{\mathrm{a}}\end{array}$ & $p$-value \\
\hline \multicolumn{4}{|l|}{ High-risk stigmata } \\
\hline Enhancing mural nodule $\geq 5 \mathrm{~mm}$ & $13(81)$ & $17(59)$ & 0.19 \\
\hline MPD size $\geq 10 \mathrm{~mm}$ & $6(38)$ & $4(14)$ & 0.13 \\
\hline \multicolumn{4}{|l|}{ Worrisome features } \\
\hline Cyst size $\geq 3 \mathrm{~cm}$ & $8(50)$ & $17(59)$ & 0.76 \\
\hline Enhancing mural nodule $<5 \mathrm{~mm}$ & 0 & 0 & $>0.99$ \\
\hline Thickened enhancing cyst wall & $9(56)$ & $17(59)$ & $>0.99$ \\
\hline MPD size $5-9 \mathrm{~mm}$ & $3(19)$ & $6(21)$ & $>0.99$ \\
\hline Abrupt MPD calibre change & $6(38)$ & $1(11)$ & 0.005 \\
\hline Lymphadenopathy & 0 & 0 & $>0.99$ \\
\hline
\end{tabular}

aNumbers in parentheses represent percentages. MPD - main pancreatic duct

perfect inter-rater agreement. Therefore, the use of LSR to detect suspicious lesions on an ADC map could contribute to improved lesion assessment. Considering the major guidelines, which recommend FNA prior to resection for determining the presence of suspicious or positive cytology $[21,23,24]$, identification of the most suspicious solid component of IPMNs is a prerequisite for selecting the appropriate FNA sampling site. LSR may act as a guide for
FNA by identifying the suspicious site for targeted biopsy, enabling endoscopists to determine the sampling site with greater confidence.

We considered that the lower AUCs for $\mathrm{ADC}_{\text {min }}$ and $\mathrm{ADC}_{\text {mean }}$ than for LSR in $2 \mathrm{D}$ analysis were related to the overlap in ADC values between IPMNs with high- and low-grade dysplasia, as previously reported by Kang et al. [10]. Therefore, it is difficult to differentiate IPMNs with 
Table 3. Comparison of the area under the receiver operating characteristic curve between IPMNs with high- and low-grade dysplasia

\begin{tabular}{|c|c|c|c|c|c|c|c|c|}
\hline & \multicolumn{2}{|c|}{$\begin{array}{l}\text { IPMNs with high-grade dysplasia } \\
\qquad n=16\end{array}$} & \multicolumn{2}{|c|}{$\begin{array}{l}\text { IPMNs with low-grade dysplasia } \\
\qquad n=29\end{array}$} & \multirow[t]{2}{*}{ AUC } & \multicolumn{2}{|c|}{$95 \% \mathrm{Cl}$} & \multirow[t]{2}{*}{$p$-value } \\
\hline & Ave & SD & Ave & SD & & Lower limit & Upper limit & \\
\hline \multicolumn{9}{|l|}{ 2D analysisa } \\
\hline $\mathrm{ROI}\left(\mathrm{mm}^{2}\right)$ & 53.81 & 11.15 & 53.93 & 13.99 & 0.50 & 0.33 & 0.68 & 0.97 \\
\hline LSR & 0.30 & 0.10 & 0.23 & 0.09 & 0.72 & 0.56 & 0.88 & 0.01 \\
\hline$A D C_{\min }$ & 1674.69 & 480.35 & 1728.30 & 843.04 & 0.60 & 0.43 & 0.77 & 0.25 \\
\hline$A D C_{\text {mean }}$ & 1989.06 & 588.52 & 2225.10 & 1010.67 & 0.64 & 0.47 & 0.81 & 0.10 \\
\hline \multicolumn{9}{|l|}{ 3D analysisa } \\
\hline VOI $\left(\mathrm{cm}^{3}\right)$ & 20.99 & 29.01 & 15.17 & 28.01 & 0.57 & 0.39 & 0.74 & 0.46 \\
\hline$A D C_{\text {overall mean }}$ & 2394.93 & 351.72 & 2859.04 & 402.10 & 0.81 & 0.68 & 0.95 & $<0.05$ \\
\hline$A D C_{\text {mean0-10 }}$ & 1561.66 & 354.26 & 2319.24 & 345.63 & 0.93 & 0.86 & 1.01 & $<0.05$ \\
\hline$A D C_{\text {mean10-25 }}$ & 1793.10 & 346.31 & 2469.09 & 342.34 & 0.91 & 0.81 & 1.00 & $<0.05$ \\
\hline$A D C_{\text {mean25-50 }}$ & 2163.44 & 345.15 & 2709.03 & 368.73 & 0.85 & 0.73 & 0.97 & $<0.05$ \\
\hline$A D C_{\text {skewness }}$ & -0.35 & 0.36 & -0.06 & 0.67 & 0.63 & 0.47 & 0.80 & 0.11 \\
\hline$A D C_{\text {kurtosis }}$ & -0.000048 & 0.88 & 0.11 & 0.75 & 0.58 & 0.39 & 0.77 & 0.40 \\
\hline$A D C_{\text {entropy }}$ & 4.11 & 0.18 & 3.85 & 0.44 & 0.72 & 0.56 & 0.87 & 0.01 \\
\hline
\end{tabular}

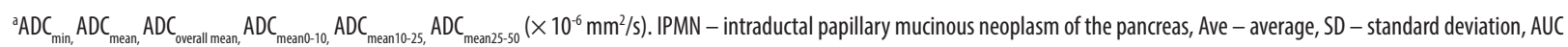
- area under the curve, $\mathrm{Cl}$ - confidence interval, ROI - region of interest, LSR - lesion-to-spinal cord signal intensity ratio, $A D C$ - apparent diffusion coefficient, VOI - volume of interest

Table 4. Results of inter-rater reliability assessment

\begin{tabular}{|c|c|c|c|c|c|}
\hline \multicolumn{2}{|l|}{ Parameter } & \multirow{3}{*}{$\begin{array}{c}\text { Intraclass correlation } \\
\text { coefficient } \\
0.259\end{array}$} & \multicolumn{2}{|c|}{$95 \% \mathrm{Cl}$} & \multirow{3}{*}{$\begin{array}{c}\text { Degree of agreement } \\
\text { Fair }\end{array}$} \\
\hline & & & \multirow{2}{*}{$\begin{array}{l}\text { Lower limit } \\
-0.034\end{array}$} & \multirow{2}{*}{$\begin{array}{c}\text { Upper limit } \\
0.511\end{array}$} & \\
\hline 2D analysis & ROl & & & & \\
\hline & LSR & 0.898 & 0.822 & 0.943 & Almost perfect \\
\hline & $A D C_{\text {min }}$ & 0.339 & 0.053 & 0.573 & Fair \\
\hline & $A D C_{\text {mean }}$ & 0.379 & 0.099 & 0.603 & Fair \\
\hline \multirow[t]{8}{*}{ 3D analysis } & VOl & 0.832 & 0.714 & 0.904 & Almost perfect \\
\hline & $A D C_{\text {overall mean }}$ & 0.697 & 0.509 & 0.821 & Substantial \\
\hline & $A D C_{\text {meano-10 }}$ & 0.399 & 0.123 & 0.618 & Fair \\
\hline & $A D C_{\text {mean10-25 }}$ & 0.219 & -0.077 & 0.479 & Fair \\
\hline & $A D C_{\text {mean25-50 }}$ & 0.563 & 0.326 & 0.734 & Moderate \\
\hline & $A D C_{\text {skewness }}$ & 0.153 & -0.144 & 0.425 & Slight \\
\hline & $A D C_{\text {kurtosis }}$ & 0.361 & 0.079 & 0.59 & Fair \\
\hline & $A D C_{\text {entropy }}$ & 0.744 & 0.579 & 0.851 & Substantial \\
\hline
\end{tabular}

C - confidence interval, ROI - region of interest, LSR - lesion-to-spinal cord signal intensity ratio, ADC - apparent diffusion coefficient, VOI - volume of interest

Table 5. Results of multivariate logistic regression analysis and comparison of diagnostic performance of continuous variables in predicting intraductal papillary mucinous neoplasm of the pancreas with high-grade dysplasia

\begin{tabular}{|c|c|c|c|c|c|c|c|c|c|}
\hline \multirow[t]{2}{*}{ Parameter } & \multicolumn{3}{|c|}{ Multivariate analysis } & \multirow{2}{*}{$\begin{array}{l}\text { Cut-off } \\
\text { value }\end{array}$} & \multicolumn{5}{|c|}{ Diagnostic performance } \\
\hline & OR & $95 \% \mathrm{Cl}$ & $p$-value & & Sensitivity & Specificity & PPV & NPV & Accuracy \\
\hline \multicolumn{10}{|l|}{ 2D analysis } \\
\hline LSR & 3.26 & $0.52-20.37$ & 0.21 & 0.25 & 69 & 69 & 55 & 80 & 69 \\
\hline \multicolumn{10}{|l|}{ 3D analysis } \\
\hline ADCoverall mean & 23.19 & $2.42-222.64$ & $<0.05$ & $2.48 \mathrm{a}$ & 75 & 83 & 71 & 86 & 80 \\
\hline ADCentropy & 20.77 & $2.11-204.30$ & $<0.05$ & 4.04 & 81 & 69 & 59 & 87 & 73 \\
\hline
\end{tabular}

IPMN - intraductal papillary mucinous neoplasm of the pancreas, LSR - lesion-to-spinal cord signal intensity ratio, ADC - apparent diffusion coefficient, PPV - positive predictive value, NPV - negative predictive values. All data for diagnostic performance, except for cut-off values, are expressed as percentages. ${ }^{a}{ }^{\circ} C\left(\times 10^{-3} \mathrm{~mm}^{2} / \mathrm{s}\right)$ 


\section{$2 \mathrm{D}$ analysis}
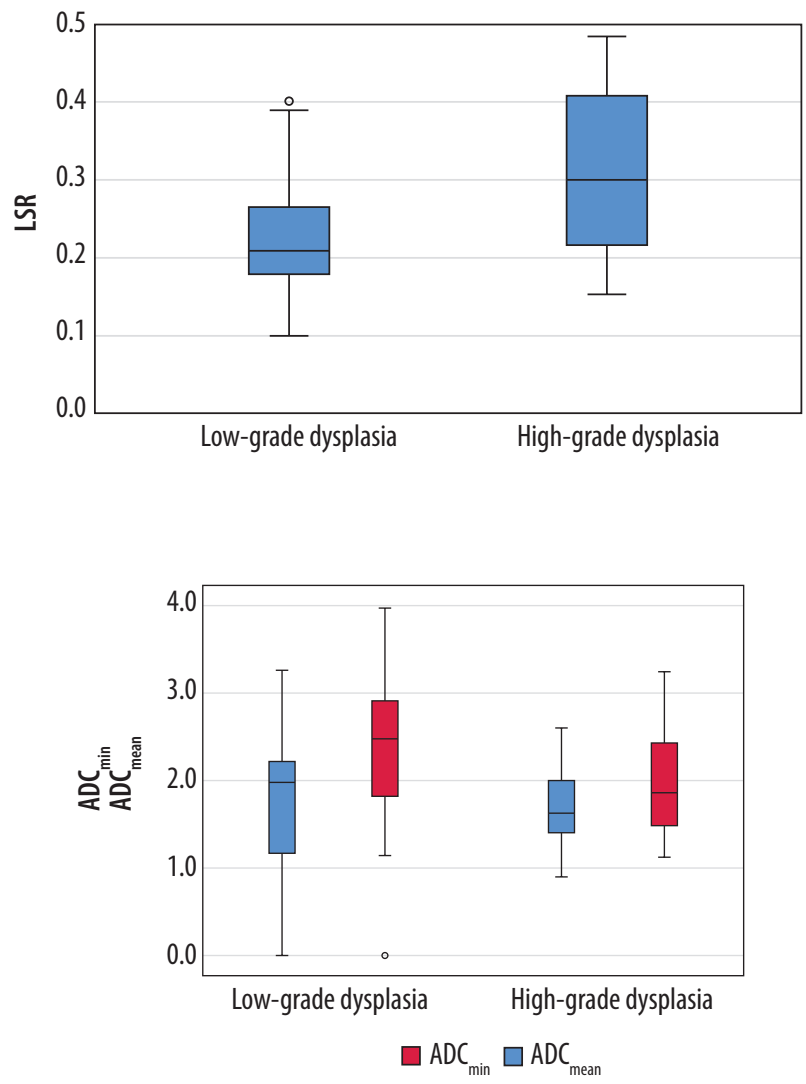

3D analysis
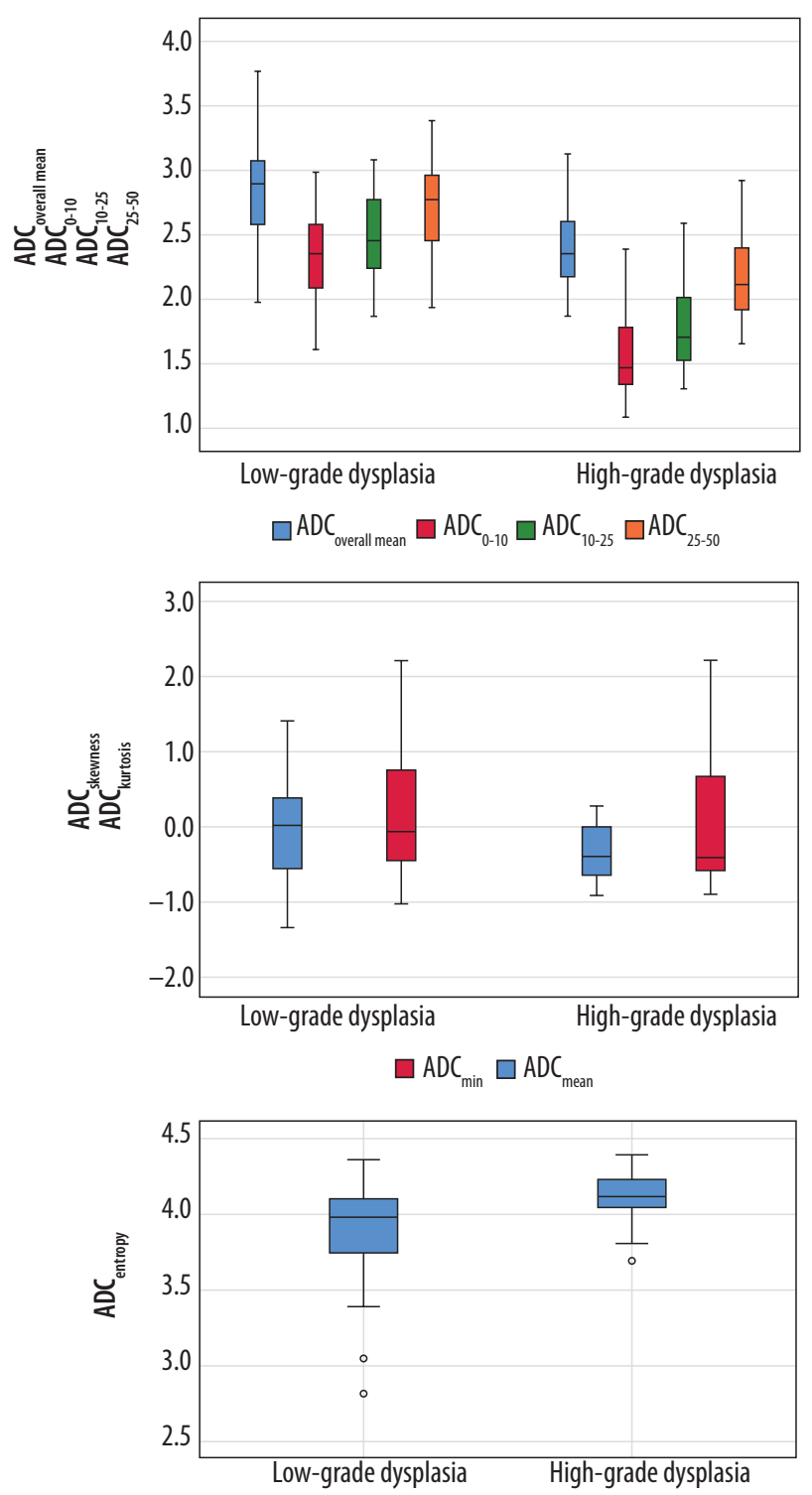

Figure 6. Box-and-whisker plots comparing quantitative variables obtained from 2D and 3D analyses of intraductal papillary mucinous neoplasm of the pancreas (IPMN) with low- and high-grade dysplasia. Circles indicate outliers. Medians (line through boxes) for apparent diffusion coefficient $(A D C)-A D C_{\text {overall mean" }} A D C_{\text {mean0-10' }} A D C_{\text {mean10-25' }} A D C_{\text {mean25-50 }}$ were lower in IPMNs with high-grade dysplasia than those in IPMNs with low-grade dysplasia. LSR - lesion-to-spinal cord signal intensity ratio

high-grade dysplasia from those with low-grade dysplasia using $\mathrm{ADC}_{\text {min }}$ and $\mathrm{ADC}_{\text {mean }}$ values obtained by $2 \mathrm{D}$ analysis, because both lesion types presumably have histopathologically similar internal structures.

However, VOIs in 3D analysis showed almost perfect inter-rater agreement, which was expected because $3 \mathrm{D}$ analysis is considered to be an objective method for highly reproducible evaluation of lesion heterogeneity. Among the $3 \mathrm{D}$ analysis parameters, $\mathrm{ADC}_{\text {overall mean }}$ and $\mathrm{ADC}_{\text {entropy }}$ showed significant between-group differences in univariate analysis and substantial inter-rater agreement. Multivariate analysis showed that $\mathrm{ADC}_{\text {overall mean }}$ and $\mathrm{ADC}_{\text {entropy }}$ were independent predictors of IPMNs with high-grade dysplasia. Malignant tumours tend to appear as heterogeneous tissue, resulting in unpredictable intensity characteristics within the tissue. Entropy is a mea- sure of uncertainty and randomness in image values [22]. IPMNs with high-grade dysplasia have a more irregular epithelial growth pattern and a disorganized polarity than those with low-grade dysplasia [3]. In this study, higher $\mathrm{ADC}_{\text {entropy }}$ was seemingly caused by this histopathological phenomenon. Hoffman et al. recently reported that $\mathrm{ADC}_{\text {entropy }}$ of $3 \mathrm{D}$ whole-lesion histogram metrics was a significant factor for differentiating benign and malignant branch-type IPMNs [20]. However, the AUC for $\mathrm{ADC}_{\text {entropy }}$ in the present study was not as high as that reported by Hoffman et al. [20], probably because of differences in the applied $b$-value [25]. In their study, DWI was performed with a high $b$-value of $500 \mathrm{~s} / \mathrm{mm}^{2}$ [20], whereas our high $b$-value was $800 \mathrm{~s} / \mathrm{mm}^{2}$. Moreover, the DWI matrix in their protocol $(128-144 \times 192)$ was larger than the one we used for 1.5 -T MRI $(77 \times 128)$. There- 
fore, the irregularity of the intra-lesion voxel distribution could be indistinct, and the possible increased overlap of $\mathrm{ADC}_{\text {entropy }}$ between IPMNs with high-grade dysplasia and IPMNs with low-grade dysplasia might have confounded our results. These factors may have contributed to our lower AUC for $\mathrm{ADC}_{\text {entropy }}$. Further studies are needed to investigate the role of $\mathrm{ADC} \mathrm{C}_{\text {entropy }}$ based on standardized MRI protocols.

This study had several limitations. First, this was a retrospective, single-centre study with a limited number of patients. Additional studies with large cohorts are needed to further investigate the technique utilized in this study. Second, clinical variables and cyst growth rates $>5 \mathrm{~mm} /$ 2 years, included as evaluation points in the revised 2017 guidelines, were not evaluated. Third, 3T-MRI has a greater magnetic susceptibility effect than does 1.5T-MRI. This increases the possibility of image distortion. Therefore, if lesions with hemosiderin deposition and the resultant susceptibility effect are present, magnetic susceptibility may affect 3D analysis. Fourth, we used ADC values with $b$-values of 50 and $800 \mathrm{~s} / \mathrm{mm}^{2}$, generated from a monoexponential model (conventional DWI). Theoretically, this method is influenced by perfusion and diffusion ef- fects. Intravoxel incoherent motion (IVIM)-DWI, which is based on a bi-exponential model with multiple $b$-value combinations, and which can separate perfusion and diffusion effects, may therefore be more valuable because it can provide more accurate data about signal decay, emphasizing perfusion or diffusion effects [26]. Further studies using IVIM-DWI are needed to investigate its potential as a quantitative biomarker for predicting malignant IPMNs.

\section{Conclusions}

The present results suggest that combining the $\mathrm{ADC}_{\text {overall }}$ mean and $\mathrm{ADC}_{\text {entropy }}$ values from 3D analysis and LSR from $2 \mathrm{D}$ analysis may assist in predicting IPMNs with highgrade dysplasia. Assessment of $\mathrm{ADC}_{\text {overall mean }}$ and $\mathrm{ADC}_{\mathrm{en} \text { - }}$ tropy from 3D analysis could help to identify patients with IPMNs with high-grade dysplasia using non-enhanced MRI techniques.

\section{Conflict of interest}

The authors report no conflict of interest.

\section{References}

1. Ohhashi K, Murakami Y, Maruyama M, et al. Four cases of mucous secreting pancreatic cancer. Prog Digest Endosc 1982; 20: 348-351.

2. Morohoshi T, Kanda M, Asanuma K, et al. Intraductal papillary neoplasms of the pancreas. A clinicopathologic study of 6 patients. Cancer 1989; 64: 1329-1335.

3. European Study Group on Cystic Tumours of the Pancreas. European evidence-based guidelines on pancreatic cystic neoplasms. Gut 2018; 67: 789-804.

4. Maire F, Hammel P, Terris B, et al. Prognosis of malignant intraductal papillary mucinous tumours of the pancreas after surgical resection. Comparison with pancreatic ductal adenocarcinoma. Gut 2000; 51: 717-722.

5. Suzuki Y, Atomi Y, Sugiyama M, et al. Cystic neoplasm of the pancreas: a Japanese multiinstitutional study of intraductal papillary mucinous tumor and mucinous cystic tumor. Pancreas 2004; 28: 241-246.

6. Shimada K, Sakamoto Y, Sano T, et al. Invasive carcinoma originating in an intraductal papillary mucinous neoplasm of the pancreas: a clinicopathologic comparison with a common type of invasive ductal carcinoma. Pancreas 2006; 32: 281-287.

7. Woo SM, Ryu JK, Lee SH, et al. Survival and prognosis of invasive intraductal papillary mucinous neoplasms of the pancreas: comparison with pancreatic ductal adenocarcinoma. Pancreas 2008; 36 : 50-55.

8. Poultsides GA, Reddy S, Cameron JL, et al. Histopathologic basis for the favorable survival after resection of intraductal papillary mucinous neoplasm- associated invasive adenocarcinoma of the pancreas. Ann Surg 2010; 251: 470-476.

9. Kim SH, Lee JM, Lee ES, et al. Intraductal papillary mucinous neoplasms of the pancreas: evaluation of malignant potential and surgical resecta- bility by using MR imaging with MR cholangiography. Radiology 2015; 274: 723-733.

10. Kang KM, Lee JM, Shin CI, et al. Added value of diffusion-weighted imaging to MR cholangiopancreatography with unenhanced MR imaging for predicting malignancy or invasiveness of intraductal papillary mucinous neoplasm of the pancreas. J Magn Reson Imaging 2013; 38: 555-563.

11. Ogawa T, Horaguchi J, Fujita N, et al. Diffusion-weighted magnetic resonance imaging for evaluating the histological degree of malignancy in patients with intraductal papillary mucinous neoplasm. J Hepatobiliary Pancreat Sci 2014; 21: 801-808.

12. Jang KM, Kim SH, Min JH, et al. Value of diffusion-weighted MRI for differentiating malignant from benign intraductal papillary mucinous neoplasms of the pancreas. AJR Am J Roentgenol 2014; 203: 992-1000.

13. Zhang L, Rao SX, Xu XF, et al. Value of apparent diffusion coefficient for predicting malignancy of intraductal papillary mucinous neoplasms of the pancreas. Diagn Interv Radiol 2016; 22: 308-313.

14. Kartalis N, Lindholm TL, Aspelin P, et al. Diffusion-weighted magnetic resonance imaging of pancreas tumours. Eur Radiol 2009; 19: 19811990.

15. Sandrasegaran K, Akisik FM, Patel AA, et al. Diffusion-weighted imaging in characterization of cystic pancreatic lesions. Clin Radiol 2011; 66: 808-814.

16. Uto T, Takehara Y, Nakamura Y, et al. Higher sensitivity and specificity for diffusion-weighted imaging of malignant lung lesions without apparent diffusion coefficient quantification. Radiology 2009; 252: 247-254.

17. Kitazume Y, Taura S, Nakaminato S, et al. Diffusion-weighted magnetic resonance imaging to differentiate malignant from benign gallbladder disorders. Eur J Radiol 2016; 85: 864-873. 
18. Çakmak V, Ufuk F, Karabulut N. Diffusion weighted MRI of pulmonary lesions: Comparison of apparent diffusion coefficient and lesion-to-spinal cord signal intensity ratio in lesion characterization. J Magn Reson Imaging 2017; 45: 845-854.

19. Igarashi T, Ashida H, Morikawa K, et al. Evaluating the malignant potential of intraductal papillary mucinous neoplasm of the pancreas: added value of non-enhanced endoscopic ultrasound to supplement non-enhanced magnetic resonance imaging. Pol J Radiol 2018; 83: e426-e436.

20. Hoffman DH, Ream JM, Hajdu CH, et al. Utility of whole-lesion ADC-histogram metrics for assessing the malignant potential of pancreatic intraductal papillary mucinous neoplasms (IPMNs). Abdom Radiol 2017; 42: 1222-1228.

21. Tanaka M, Fernández-del Castillo C, Kamisawa T, et al. Revisions of international consensus Fukuoka guidelines for the management of IPMN of the pancreas. Pancreatology 2017; 17: 738-753.

22. Just $\mathrm{N}$. Improving tumour heterogeneity MRI assessment with histograms. Br J Cancer 2014; 111: 2205-2213.

23. Vege SS, Ziring B, Jain R, et al.; Clinical Guidelines Committee, American Gastroenterology Association. American gastroenterological association institute guideline on the diagnosis and management of asymptomatic neoplastic pancreatic cysts. Gastroenterology 2015; 148: 819-822.

24. Berland L, Silverman SG, Gore R, et al. Managing incidental findings on abdominal CT: white paper of the ACR incidental findings committee. J Am Coll Radiol 2010; 7: 754-773.

25. Bougias H, Ghiatas A, Priovolos D, et al. Whole-lesion apparent diffusion coefficient (ADC) metrics as a marker of breast tumour characterization-comparison between ADC value and ADC entropy. Br J Radiol 2016; 89: 20160304.

26. Le Bihan D, Breton E, Lallemand D, et al. MR imaging of intravoxel incoherent motions: application to diffusion and perfusion in neurologic disorders. Radiology 1986; 161 :401-407. 